\title{
On coincidence problem in ELKO dark energy model
}

\author{
H. Mohseni Sadjadi* \\ Department of Physics, University of Tehran, \\ P. O. B. 14395-547, Tehran 14399-55961, Iran
}

June 19, 2018

\begin{abstract}
We study the critical points of a universe dominated by ELKO spinor field dark energy and a barotropic matter without considering a specific potential or interaction. The coincidence problem and attractor solutions are discussed at late time, and it is shown that the coincidence problem can not be solved in this model.
\end{abstract}

PACS numbers: 95.36.+x, 95.35.+d, 98.80.-k

\section{Introduction}

To describe the present accelerated expansion of the universe 11, many models have been considered. In dark energy models, almost $70 \%$ of our universe is assumed to be filled with a smooth unknown matter with negative pressure known as dark energy. The first candidate for dark energy was the cosmological constant which may arise from quantum vacuum energy density [2]. Other candidates proposed for dark energy were exotic dynamical scalar fields such as quintessence or phantom, but, spinor dark energy model has also attracted some attentions recently [3].

In [4], a class of non standard spinors, constructed in momentum space from the eigenspinors of the charge conjugation operator, known as ELKO spinor(Eigenspinoren des LadungsKonjugationsOperators), satisfying $(C P T)^{2}=$ -1 , was introduced and proposed as dark matter candidate . Cosmological and gravitational consequences of this model were studied in [5].

Although, at the beginning, ELKO spinor was contemplated as dark matter, but subsequently, it was considered as a potential candidate for inflation [6], or present acceleration of the universe [7]. This last point is the subject of the present paper.

*mohsenisad@ut.ac.ir 
Viable models must be consistent with astrophysical data. These data indicate that, despite the expansion of the universe, the ratio of matter to dark energy density is of order $r:=\frac{\rho_{m}}{\rho_{d}} \simeq \mathcal{O}(1)$. This problem, i.e. why dark energy density $\left(\rho_{d}\right)$ is of the same order of the matter density $\left(\rho_{m}\right)$, is known as the coincidence problem [8].

In [9], it was found that, for some special potentials and also for some special interactions between (dark) matter and dark energy, the coincidence problem cannot be solved in ELKO dark energy model.

In this paper, we consider the late time evolution of the universe, which is assumed to be almost composed of a barotropic matter and ELKO dark energy. By considering a general dark energy potential and also a general interaction between matter and dark energy, it is shown that, in principle, the coincidence problem cannot be alleviated in this model.

We use the units $\hbar=c=1$.

\section{Attractor solutions in ELKO cosmology}

ELKO model in a curved space is characterized by the action

$$
S=\int\left[\frac{1}{2} \vec{\psi} \overleftarrow{\nabla_{\nu}} \nabla^{\nu} \psi+V(\vec{\psi} \psi)\right] \sqrt{-g} d^{4} x
$$

where $g$ is determinant of the metric tensor, $V$ is the potential, and $\vec{\psi}$ is dual spinor. $\nabla_{\nu}$ are covariant derivative components acting on spinors as

$$
\nabla_{\nu} \psi=\left(\partial_{\nu}-\Gamma_{\nu}\right) \psi
$$

where $\Gamma_{\nu}=\frac{i}{4} \omega_{\nu a b} \sigma^{a b} . \omega_{\nu a b}$ are spin connection components, and in terms of Dirac matrices we have $\sigma^{a b}=\frac{i}{2}\left[\gamma^{a}, \gamma^{b}\right]$. The energy momentum tensor is obtained as (the derivation of energy momentum tensor of ELKO spinors can be found, in details, in [5])

$$
\begin{aligned}
& T^{\mu \nu} \neg \overleftarrow{\psi} \overleftarrow{\nabla^{(\mu}} \nabla^{\nu)} \psi-g^{\mu \nu}\left[\frac{1}{2} \vec{\psi} \overleftarrow{\nabla_{\alpha}} \nabla^{\alpha} \psi-V(\vec{\psi} \psi)\right] \\
& -\frac{i}{4} \nabla_{\beta}\left[\bar{\psi} \overleftarrow{\nabla}\left(\mu \sigma^{\nu) \beta} \psi+\vec{\psi} \sigma^{\beta(\mu} \nabla^{\nu)} \psi\right] .\right.
\end{aligned}
$$

In the following we consider the spatially flat FRW space-time

$$
d s^{2}=d t^{2}-a^{2}(t)\left(d x^{2}+d y^{2}+d z^{2}\right),
$$

where $a(t)$ is the scale factor. In this background by writing ELKO spinor as $\psi=\phi \lambda$, where $\lambda$ is a constant spinor satisfying $\vec{\lambda} \lambda=1$ [5], the energy 
momentum tensor may be derived as

$$
\begin{aligned}
& T_{0}^{0}=\rho_{d}=\frac{1}{2} \dot{\phi}^{2}+V+\frac{3}{8} H^{2} \phi^{2} \\
& T_{j}^{i}=-\delta_{j}^{i} P_{d}=\delta_{j}^{i}\left(\frac{3}{8} H^{2} \phi^{2}+V-\frac{1}{2} \dot{\phi}^{2}+\frac{1}{4} \dot{H} \phi^{2}+\frac{1}{2} \phi \dot{\phi} H\right) .
\end{aligned}
$$

$\rho_{d}$, and $P_{d}$ are the energy density and pressure of dark energy respectively. In the absence of interaction, the continuity equation for the dark sector

$$
\dot{\rho}_{d}+3 H\left(P_{d}+\rho_{d}\right)=0,
$$

implies

$$
\ddot{\phi}+3 H \dot{\phi}+\frac{d V}{d \phi}-\frac{3}{4} H^{2} \phi=0 .
$$

Now let us consider a FRW Universe dominated by ELKO spinor dark energy and a barotropic matter $\rho_{m}$ whose the pressure is $P_{m}=w_{m} \rho_{m}$. The equation of state parameter of the matter, $w_{m}$, is assumed to be non negative $w_{m} \geq 0$, e.g. for cold dark matter we have $w_{m}=0$. The continuity equations for dark energy and matter component in the presence of the interaction source $C$ become

$$
\begin{aligned}
& \dot{\rho_{d}}+3 H\left(P_{d}+\rho_{d}\right)=-C \\
& \dot{\rho_{m}}+3 H \gamma \rho_{m}=C,
\end{aligned}
$$

where $\gamma:=w_{m}+1$. The Friedmann equation is given by

$$
H^{2}=\frac{1}{3 M_{p}^{2}}\left(\rho_{d}+\rho_{m}\right)
$$

which can be rewritten as

$$
\left(1-\frac{1}{8 M_{p}^{2}} \phi^{2}\right) H^{2}=\frac{1}{3 M_{p}^{2}}\left(\rho_{m}+\frac{1}{2} \dot{\phi}^{2}+V\right) .
$$

$M_{p}$ is the reduced Planck mass. So the effective gravitational coupling constant is modified in this theory. For $\left(\rho_{m}+\frac{1}{2} \dot{\phi}^{2}+V\right)>0$, we must have $|\phi|<2 \sqrt{2} M_{p}$.

Raychaudhuri equation reads:

$$
\left(1-\frac{1}{8 M_{p}^{2}} \phi^{2}\right) \dot{H}=-\frac{1}{2 M_{p}^{2}}\left(\gamma \rho_{m}+\dot{\phi}^{2}-\frac{1}{2} H \phi \dot{\phi}\right) .
$$

The scalar field, $\phi$, satisfies the classical equation of motion:

$$
\dot{\phi}\left(\ddot{\phi}+3 H \dot{\phi}+V_{, \phi}-\frac{3}{4} H^{2} \phi\right)=-C .
$$


The equation of state parameter of the Universe defined by $w=\frac{P_{d}+P_{m}}{\rho_{d}+\rho_{m}}$ is given by $w=-1+\frac{2}{3} \omega$, where $\omega=-\frac{\dot{H}}{H^{2}}$.

To study the cosmological dynamics of this model, we define dimensionless variables [10]

$$
x=\frac{\dot{\phi}}{\sqrt{6} M_{p} H}, \quad y=\frac{\sqrt{V}}{\sqrt{3} M_{p} H}, \quad z=\frac{\sqrt{\rho_{m}}}{\sqrt{3} M_{p} H}, u=\frac{\phi}{M_{p} \sqrt{8}} .
$$

Hence

$$
\left(1-u^{2}\right) \omega=3 x^{2}+1.5 \gamma z^{2}-\sqrt{3} x u .
$$

By the assumption that the potential is only a function of $u$, and by defining

$$
f(u)=\frac{M_{p} V_{, \phi}}{V}
$$

where $V_{, \phi}=\frac{d V}{d \phi}$, we find out the autonomous system of differential equations

$$
\begin{aligned}
x^{\prime} & =(\omega-3) x+\frac{\sqrt{3}}{2} u-\sqrt{\frac{3}{2}} y^{2} f-C_{1} \\
y^{\prime} & =\left(\sqrt{\frac{3}{2}} x f+\omega\right) y \\
z^{\prime} & =\left(\omega-\frac{3}{2} \gamma\right) z+C_{2} \\
u^{\prime} & =\frac{\sqrt{3}}{2} x
\end{aligned}
$$

where, prime denotes derivatives with respect to the e-folding time $\mathcal{N}=\ln a$, and

$$
\begin{aligned}
C_{1} & =\frac{C}{\sqrt{6} M_{p} H^{2} \dot{\phi}} \\
C_{2} & =\frac{C}{2 \sqrt{3} M_{p} H^{2} \sqrt{\rho_{m}}}=\frac{x}{z} C_{1} .
\end{aligned}
$$

$C$ is taken to be a function of $x, z, u$. For the sake of generality, we do not restrict ourselves to a specific interaction.

Note that $x, y, z, u$ are not independent and are constraint to the Friedmann equation :

$$
x^{2}+y^{2}+z^{2}+u^{2}=1 .
$$

Most generally, critical points of the autonomous system (16) denoted with $\{\bar{x}, \bar{y}, \bar{z}, \bar{u}\}$ can be arranged as follows:

I: $\{\bar{x}=0, \bar{y}=0, \bar{z}=0, \bar{u}=0\}$ which is in contradiction with (18), and then is ruled out.

II: $\{\bar{x}=0, \bar{y}=0, \bar{z} \neq 0, \bar{u}=0\}$. From (18) we have $\bar{z}^{2}=1$. (16) implies $\bar{C}_{2}=0$ and $\bar{C}_{1}=0$, where bar denotes the value at the critical point. 
III: $\{\bar{x}=0, \bar{y}=0, \bar{z}=0, \bar{u} \neq 0\}$. From (18) we have $\bar{u}^{2}=1$, (16) gives $\bar{C}_{2}=0$ and $\bar{C}_{1}= \pm \frac{\sqrt{3}}{2}$.

$\mathrm{IV}:\{\bar{x}=0, \bar{y}=0, \bar{z} \neq 0, \bar{u} \neq 0\}$. In this case, $\bar{u}^{2}+\bar{z}^{2}=1$, and $\bar{C}_{1}=\frac{\sqrt{3}}{2} \bar{u}$. (14) gives $\bar{\omega}=\frac{3}{2} \gamma$. We have also $\bar{C}_{2}=0$.

It is obvious that in the absence of interaction critical points III and IV do not exist.

$\mathrm{V}:\{\bar{x}=0, \bar{y} \neq 0, \bar{z}=0, \bar{u}=0\}, \bar{y}^{2}=1$. From (16) we have $\bar{C}_{1}=-\sqrt{\frac{3}{2}} \bar{f}$, $\bar{C}_{2}=0$, and (14) gives $\bar{\omega}=0$.

$\mathrm{VI}:\{\bar{x}=0, \bar{y} \neq 0, \bar{z} \neq 0, \bar{u}=0\}, \bar{z}^{2}+\bar{y}^{2}=1$. From (16) we obtain $\bar{\omega}=0$ which using $\bar{\omega}=1.5 \gamma \bar{z}^{2}$, results in $\gamma=0$. In this case, $\bar{C}_{1}=-\sqrt{\frac{3}{2}} \bar{f} \bar{y}^{2}$, and $\bar{C}_{2}=0$.

In the absence of interaction, critical points $\mathrm{V}$ and VI exist only for potential satisfying $\bar{f}(u)=f(\bar{u})=0$. E.g. for the potential $V \propto \exp (\lambda \phi)$, we have $\bar{f}(u)=\lambda M_{P}$, and these critical points do not exist when $C=0$.

VII: $\{\bar{x}=0, \bar{y} \neq 0, \bar{z}=0, \bar{u} \neq 0\}, \bar{u}^{2}+\bar{y}^{2}=1$. From (16) we obtain $\bar{C}_{2}=0, \bar{\omega}=0$ and $\bar{C}_{1}=\frac{\sqrt{3}}{2} \bar{u}-\sqrt{\frac{3}{2}} \bar{y}^{2} \bar{f}$.

VIII: $\{\bar{x}=0, \bar{y} \neq 0, \bar{z} \neq 0, \bar{u} \neq 0\} . \quad \bar{\omega} \bar{y}=0$ gives $\bar{\omega}=0$. We obtain also $\bar{C}_{2}=\frac{3}{2} \gamma \bar{z}$, and $\bar{C}_{1}=\frac{\sqrt{3}}{2} \bar{u}-\sqrt{\frac{3}{2}} \bar{y}^{2} \bar{f}$. Using $C_{2}=\frac{x}{z} C_{1}$, we obtain $\bar{C}_{2}=0$ which results in $\gamma=0$. This could be obtained in another way: as $\bar{\omega}=\frac{3 \gamma}{2} \frac{\bar{z}^{2}}{1-\bar{u}^{2}}, \bar{\omega}=0$ implies $\gamma=0$. In the absence of interaction, critical points VII and VIII exist only for potential satisfying $\bar{u}=\sqrt{2} \bar{y}^{2} \bar{f}$.

All the critical points are characterized by $\bar{x}=0$, so as it can be seen from eqs.(17) and (12), $C_{1}$ may be singular or not generally well defined. In theses cases the attractor solutions do not exist. Instead, if the order of magnitude of numerator of $C_{1}$ in (17) (i.e. $C$ ) is less or equal than that of its denominator at a critical point, $C_{1}$ is still finite and well defined. To elucidate this subject, let us consider interactions $C^{I}=\sigma H \rho_{m}$ and $C^{I I}=$ $\varsigma H\left(\rho_{m}+\rho_{d}\right)$, where $\sigma$ and $\varsigma$ are two constants. In terms of variables defined in (13), $C_{1}$ can be rewritten as $C_{1}^{I}=\frac{\sigma}{2} \frac{z^{2}}{x}$, which is not well defined, and $C_{1}^{I I}=\frac{\varsigma}{2 x}$ which is singular at $\bar{x}=0$. Instead, for the interaction $C^{I I I}=$ $\alpha \frac{\rho_{m}}{M_{p}} \dot{\phi}$, we have $C_{1}^{I I I}=\sqrt{\frac{3}{2}} \alpha z^{2}$ which is well defined. If one adopts the interaction $C^{I I I}$, then, critical points $I V, V, V I, V I I, V I I I$ are acceptable provided that, $\sqrt{2} \alpha \bar{z}^{2}=\bar{u}, \bar{f}=0,-\bar{f}=\alpha \bar{z}^{2}, \alpha \bar{z}^{2}=\frac{1}{\sqrt{2}} \bar{u}-\bar{y}^{2} \bar{f}$, and $\alpha \bar{z}^{2}=\frac{1}{\sqrt{2}} \bar{u}-\bar{y}^{2} \bar{f}$, hold respectively.

To study the stability of the system around the critical points, $I I-V I I I$ (if exist), we consider small perturbation around these points, $\{\bar{x}, \bar{y}, \bar{z}, \bar{u}\} \rightarrow$ $\{\bar{x}+\delta x, \bar{y}+\delta y, \bar{z}+\delta z, \bar{u}+\delta u\}$. If the real part of all the eigenvalues of $\mathcal{M}$ 
defined by

$$
\frac{d}{d \mathcal{N}}\left(\begin{array}{l}
\delta x \\
\delta y \\
\delta u
\end{array}\right)=\mathcal{M}\left(\begin{array}{l}
\delta x \\
\delta y \\
\delta u
\end{array}\right)
$$

are negative at a critical point, the system has stable attractor solution. In our model

$\mathcal{M}=\left(\begin{array}{ccc}\bar{\omega}-3-\bar{C}_{1, x} & -\sqrt{6} \bar{y} \bar{f}-\frac{\bar{y} \bar{C}_{1, z}}{\bar{z}} & \frac{\sqrt{3}}{2}-\sqrt{\frac{3}{2}} \bar{y}^{2} \frac{\overline{d f}}{d u}-\bar{C}_{1, u}-\frac{\bar{u} \bar{C}_{1, z}}{\bar{z}} \\ \sqrt{\frac{3}{2}} \bar{y} \bar{f}-\frac{\bar{y}}{1-\bar{u}^{2}} \sqrt{3} \bar{u} & \bar{\omega}-\frac{3 \gamma \bar{y}^{2}}{1-\bar{u}^{2}} & \frac{\bar{y}}{1-\bar{u}^{2}}(2 \bar{\omega} \bar{u}-3 \gamma \bar{u}) \\ \frac{\sqrt{3}}{2} & 0 & 0\end{array}\right)$.

As we are interested to study the coincidence problem, among the situations I-VIII, we need only consider cases where $r$ is of order $\mathcal{O}(1)$ or more precisely:

$$
r:=\frac{\rho_{m}}{\rho_{d}}=\frac{z^{2}}{1-z^{2}} \simeq \frac{3}{7} .
$$

In the cases III, V, and VII, we have $\bar{r}=0$, and in the case II, $\bar{r} \rightarrow \infty$. Therefore among all the possible critical points $I I-V I I I$, only IV, VI, and VIII may be consistent with $\bar{r} \sim \mathcal{O}(1)$.

In VI, and VIII, we have $\gamma=0$ which implies $w_{m}=-1$, in contradiction with our assumption that the universe is dominated by ELKO dark energy and a matter with non-negative pressure. So, finally, we are left only with the case IV. In this case

$$
\mathcal{M}_{I V}=\left(\begin{array}{ccc}
\frac{3}{2}(\gamma-2)-\bar{C}_{1, x} & 0 & \frac{\sqrt{3}}{2}-\bar{C}_{1, u}-\frac{\bar{u} \bar{C}_{1, z}}{\bar{z}} \\
0 & \frac{3}{2} \gamma & 0 \\
\frac{\sqrt{3}}{2} & 0 & 0
\end{array}\right) .
$$

One of the eigenvalues of $\mathcal{M}_{I V}$ is $\lambda=\frac{3 \gamma}{2}$ which is positive, so even in this situation the system is not stable and there is no scaling attractor. Besides for an accelerated expanding universe we must have $w<-\frac{1}{3}$, implying $\omega<1$, but in IV, we have $\bar{\omega}=\frac{3}{2} \gamma>1$ which, in contradiction with the nowadays accelerated expansion of the Universe, describes a decelerated expanding Universe.

\section{Summary}

After the introduction of ELKO spinor as a potential candidate of dark matter in [4, some attempts has been done to consider this kind of spinors as inflaton [6], and dark energy [7].

In this paper we examined this theory in the context of dark energy model, and using Friedmann and Raychaudhury equations we obtained an 
autonomous dynamical system describing the behavior of a spatially flat FRW Universe dominated by ELKO non standard spinor dark energy, interacting with a barotropic matter, at late time. We did not restrict the problem to special potentials or interactions. The critical points and attractor solutions of the problem were studied. The coincidence problem was discussed in this framework and it was found that there is no stable solution which can alleviate the coincidence problem.

\section{References}

[1] S. Perlmutter et al., Nature (London) 391, 51 (1998); A. G. Riess et al. (Supernova Search Team Collaboration), Astron. J. 116, 1009 (1998); Astron. J. 117, 707 (1999); S. Perlmutter et al. (Supernova Cosmology Project Collaboration), Astrophys. J. 517, 565 (1999); E. V. Linder, arXiv:1009.1411v2 [astro-ph.CO]; E. Elizalde, S. Nojiri, S. D. Odintsov, L. Sebastiani, and S. Zerbini, Phys. Rev. D 83, 086006 (2011); Y. L. Bolotin, O. A. Lemets, and D. A. Yerokhin, arXiv:1108.0203v3 [astroph.CO]; A. Barreira and P.P. Avelino, Phys. Rev. D 83, 103001 (2011).

[2] S. Weinberg, Rev. Mod. Phys. 61, 1 (1989).

[3] X. Zhang, H. Li, Y. Piao, and X. Zhang, Mod. Phys. Lett. A 21, 231 (2006); C. G. Boehmer, AnnalenPhys. 16, 325 (2007); Y. Cai and J. Wang, Class. Quant. Grav. 25, 165014 (2008); B. Saha, arXiv:1103.2890v1 [gr-qc]; C. G. Boehmer and J. Burnett, Mod. Phys. Lett. A 25, 101 (2010).

[4] D. V. Ahluwalia, and D. Grumiller, JCAP 07 (2005) 012, arXiv:hep-th/0412080v3; D. V. Ahluwalia and D. Grumiller, Phys. Rev. D 72, 0677701 (2005), arXiv:hep-th/0410192v2; D. V. Ahluwalia, Ch. Lee, and D. Schritt, Phys. Lett. B 687, 248 (2010); D. V. Ahluwalia, Ch. Lee, and D. Schritt, Phys. Rev. D 83, 065017 (2011).

[5] Ch. G. Boehmer, J. Burnett, D. F. Mota, and D. J. Shaw, JHEP 07(2010)053, arXiv:1003.3858v2 [hep-th]; L. Fabbri, Phys. Rev. D 85, 047502 (2012); L. Fabbri and S. Vignolo, Annalen Phys. 524, 77 (2012); G. Chee, arXiv:1007.0554v1 [gr-qc].

[6] Ch. G. Boehmer, Phys. Rev. D77, 123535 (2008); D. Gredat and S. Shankaranarayanan, JCAP 1001, 008 (2010), arXiv:0807.3336v2 [astro$\mathrm{ph}]$.

[7] C. G. Boehmer and J. Burnett, Mod. Phys. Lett. A 25, 101 (2010), arXiv:0906.1351 v1 [gr-qc]. 
[8] P. J. Steinhardt, in Critical Problems in Physics, Eds. V.L. Fitch, D. R. Marlow, and. M. A. E. Dementi, Princeton University Press, 1997; N. Straumann, arXiv:astro-ph/0009386v1; D. T. Valentini, L. Amendola, Phys. Rev. D 65, 063508 (2002); V. Sahni, Lect. NotesPhys. 653, 141 (2004); S. Nojiri and S. D. Odintsov, Gen. Rel. Grav. 38, 1285 (2006); L. Amendola, S. Tsujikawa, and M. Sami, Phys. Lett. B 632, 155 (2006); S. Nojiri and S. D. Odintsov, Phys. Lett. B 637, 139 (2006); H. M. Sadjadi and M. Alimohammadi, Phys. Rev. D 74, 103007 (2006); H. M. Sadjadi and M. Honardoost, Phys. Lett. B 647, 231 (2007); E. V. Linder and R. J. Scherrer, arXiv:0811.2797v1 [astro-ph]; M. Jamil, F. Rahaman and M. Kalam, Eur. Phys. J. C 60, 149 (2009); N. Cruz, S. Lepe, and F. Pena, Phys. Lett. B 699, 135 (2011); Ch. G. Boehmer, G CalderaCabral, N. Chan, R. Lazkoz, and R. Maartens, Phys. Rev. D 81, 083003 (2010); Ch. Ahn, Ch. Kim, and E. V. Linder, arXiv:1106.1435v1 [astroph.CO].

[9] H. Wei, Phys. Lett. B 695, 307 (2011), arXiv:1002.4230v3 [gr-qc].

[10] E. J Copeland, A. R. Liddle, and D. Wands Phys. Rev. D 57, 4686 (1998); L. Amendola, Phys. Rev. D 60, 043501 (1999); L. Amendola, C. Quercellini, D. Tocchini-Valentini, and A. Pasqui, Astrophys. J. 583, L53 (2003); M. Alimohammadi and H. M. Sadjadi, Phys. Rev. D 73,083527 (2006). 